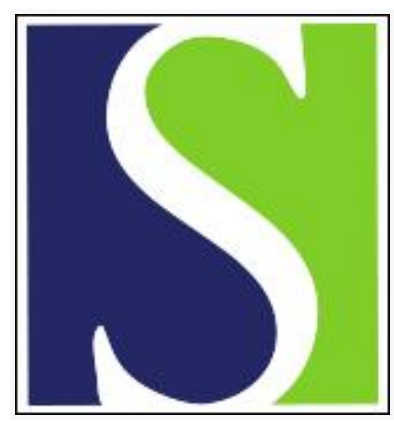

Scand J Work Environ Health 2008;34(3):198-205

https://doi.org/10.5271/sjweh.1228

Issue date: 30 Jun 2008

Effects of shift rotation and the flexibility of a shift system on daytime alertness and cardiovascular risk factors

by Viitasalo K, Kuosma E, Laitinen J, Härmä M

Affiliation: Finnair Health Services, Finnair PIc, IF/67, Fl-01053 Finnair, Finland. katriina.viitasalo@finnair.com

Refers to the following texts of the Journal: 2006;32(6):482-492

2007;33(1):51-57 2001;27(2):87-96 2006;32(6):502-514

1999;25(4):376-381 2007;33(1):45-50 1999;25(2):85-99

1997;23(4):257-265 2005;31(1):30-35

The following articles refer to this text: 2009;35(3):193-202;

2010;36(2):121-133; 2010;36(2):81-84; 2010;36(6):515-516;

2012;38(4):343-348; 2012;38(4):299-313; 2012;38(4):291-297;

2012;38(4):327-336; 2014;40(6):543-556; 2018;44(4):394-402

Key terms: blood pressure; cardiovascular risk factor; daytime alertness; flexibility; health behavior; heart rate; lipids; shift rotation; shift scheduling; shift system; shift work; sleepiness

This article in PubMed: www.ncbi.nlm.nih.gov/pubmed/18728909

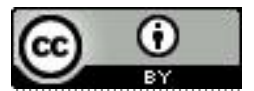




\title{
Effects of shift rotation and the flexibility of a shift system on daytime alertness and cardiovascular risk factors
}

\author{
by Katriina Viitasalo, MD, ${ }^{1}$ Eeva Kuosma, MSc, ${ }^{2}$ Jaana Laitinen, PhD, ${ }^{3}$ Mikko Härmä, MD ${ }^{4}$
}

\begin{abstract}
Viitasalo K, Kuosma E, Laitinen J, Härmä M. Effects of shift rotation and the flexibility of a shift system on daytime alertness and cardiovascular risk factors. Scand J Work Environ Health. 2008;34(34):198-205.

Objectives A controlled intervention study was conducted to evaluate the effects of two changes in shift characteristics on alertness and cardiovascular risk factors: a change in shift rotation (direction and speed) and a change in the flexibility of the shift system.

Methods Altogether 84 male workers currently working in a backward-rotating shift system volunteered for the study. A total of 40 men changed to a rapidly forward-rotating shift system, 22 changed to a more flexible shift system, and 22 remained with the old shift system. Health effects were studied with the use of clinical measurements, blood tests, and questionnaires before and after the shift changes. Analyses of variance were used with repeated measures to study associations of cardiovascular risk factors and daytime sleepiness according to the change in shift systems.

Results The mean number of days on which the workers reported sleepiness decreased in the group with the forward-rotating shift system when compared with that of the group on the old shift system (from 2.9 to 2.1 days/week, $\mathrm{P}=0.02$ ). Systolic blood pressure decreased (from 142 to $136 \mathrm{~mm} \mathrm{Hg}, \mathrm{P}=0.049$ ), and heart rate showed a declining trend (from 66 to 60 beats/minute, $\mathrm{P}=0.06$ ) in the flexible shift system when the three groups were compared.

Conclusions The study indicates that a faster speed, together with a change to the forward direction, in shift rotation alleviates daytime sleepiness. Combining individual flexibility with company-based flexibility in a shift system may have favorable effects on shift workers' blood pressure.
\end{abstract}

Key terms blood pressure; health behavior; heart rate; lipids; shift scheduling; shift work; sleepiness.

Shift work is related to disturbed sleep-wakefulness and a higher risk of cardiovascular disease (1-5). The prevalence of risk factors for coronary heart disease is higher among shift workers (6), and moreover people with coronary risk factors are more likely to be affected by rotating shift work (4). Shift work has been shown to be related to higher levels of triglyceride and lower levels of high-density lipoprotein (HDL) cholesterol (7-9), but the evidence for the relationship between shift work and hypertension or elevated blood pressure is inconclusive $(6,7,10-13)$. It has been proposed that a stressful psychosocial work environment acts as a mediator between the adverse health effects of shift work and hypertension (14). In a 10-year longitudinal Japanese study, shift work was shown to be an independent risk factor for the progression of hypertension
(15). Blood pressure has been shown to be positively related to mortality from stroke (16), and, in a Finnish epidemiologic study, mortality due to ischemic stroke was increased among shift workers (17).

Although the relationship between shift work and health is well established (18), there is no conclusive evidence of the effect of specific shift rotation systems on short- or long-term health $(18,19)$. With reference to cardiovascular health, in one short-term intervention trial, serum levels of triglyceride and glucose decreased during clockwise shift rotation as compared with during counter-clockwise shift rotation, and systolic blood pressure was lower after the clockwise rotation period (20). Another intervention study that lasted 6 months found increased ergonomic changes in shift scheduling to be associated with lower low-density lipoprotein (LDL)

1 Finnair Health Services, Vantaa, Finland.

2 Finnish Institute of Occupational Health, Centre of Expertise on Good Practices and Competence, Helsinki, Finland.

3 Finnish Institute of Occupational Health, Centre of Expertise on Health and Work Ability, Oulu, Finland.

$4 \quad$ Finnish Institute of Occupational Health, Centre of Expertise on Human Factors at Work, Helsinki, Finland.

Reprint requests to: Katriina Viitasalo, Finnair Health Services, Finnair Plc, IF/67, FI-01053 Finnair, Finland. [E-mail: katriina. viitasalo@finnair.com] 
cholesterol levels, a lower total-to-HDL cholesterol ratio, and higher HDL cholesterol levels (21).

There is a lack of intervention studies on the relationship between specific shift characteristics and fatigue or sleep-alertness (19). In general, rapidly forward-rotating shift systems seem to improve alertness and performance at work and decrease insomnia (22-24). Individual flexibility with respect to workhours is related to improved subjective health and reduced sickness absence $(21,25$, 26). However, we are not aware of earlier studies on the effects of flexible shift systems on cardiovascular risk factors or objective health.

The aim of our study was to evaluate the effects of two separate changes in shift characteristics on alertness and both behavioral and clinical risk factors for cardiovascular disease (CVD). The changes examined were a change in shift rotation (direction and speed) and a change in the flexibility (a combination of individual and company-based flexibility) of the shift system.

\section{Study population and methods}

\section{Study design}

A controlled intervention study was carried out in a line maintenance unit of an airline company. The effects of two different new shift systems on health were studied by comparing health outcomes 5 to 6 months before (October-November 2004) and 7 to 8 months after (November-December 2005) the implementation of the new shift systems. At baseline, all of the participants had been working on the old schedule for at least 18 months. The data on health outcomes were collected in the same season of the year at baseline and at the end of the follow-up to diminish the effect of annual variation in blood lipids (27) and lifestyle factors, such as dietary habits and leisure-time physical activity.

\section{Shift systems}

The line maintenance unit is responsible for small and medium-sized maintenance duties of the airline company at the Helsinki-Vantaa airport, Finland. The order of the shifts in the old schedule was EEE -- MMM -NNN -- $(E=$ evening shift, $\mathrm{M}=$ morning shift, $\mathrm{N}=$ night shift, - = day off). The shift changing times were at 0700, 1500 , and 2300, and all of the shifts were 8 hours long. One of the two new shift systems rotated very rapidly forward with a shift order of MEN--, the morning shift being from 0600 to 1600 (10 hours), the evening shift being from 1500 to 0100 (10 hours), and the night shift being from 2100 to 0600 (9 hours).

The other new shift system was called a "flexible" shift system. In this system rosters were issued 4 weeks in advance, after which the employer could only make changes in the rotas of the third or fourth week in case of changed operational needs. Changes in the rotas of the first 2 weeks were not possible without negotiation with the workers and without compensation. On the basis of mutual consent, the employer tried to fulfill the employees' wishes and needs regarding shift changes, holidays, or days off. The workers were therefore allowed some individual flexibility and control over their workhours in exchange for variability.

In the flexible shift system, the direction of rotation and the order of the shifts were basically the same as in the old shift system, but there were generally three consecutive days "off" between the evening, morning, and night shifts instead of two as in the old system. The morning shift was defined to last 10 hours from 0600 to 1600 or 11 hours from 0800 to 1900 , but in practice the duration of shift could vary from 10 to 13 hours between 0600 and 1900, depending on operational needs. The evening shift was defined as 9.5 hours from 1400 to 2330, but could vary from 6 to 13 hours between 1300 and 0200 for the same reason. The night shift was always 7 hours long from 2330 to 0630 . The planned weekly worktime varied from 30 to 36.5 hours and was balanced over a period of 4 months. The actual mean worktime per week was 32.8 hours in the flexible shift system as compared with 33.4 hours in the other two shift systems. The number of days worked per week varied from four to five in the flexible shift system, being four in the rapidly forward-rotating shift system and five in the old shift system. The period from the end of the last morning shift to the beginning of the first night shift varied from 52.5 to 80.5 hours in the flexible shift system as compared with 55 hours in the old shift system and 53 hours in the rapidly forward-rotating shift system.

The employer and the representatives of the employees wanted the workers to self-select their new shift systems, and it was therefore not possible to randomize the allocation of the participants into the study groups. Workers over 45 years of age were given priority in the selection of employees for the rapidly forward-rotating shift system because an earlier intervention study completed in the line maintenance unit had shown that this shift system had positive effects on sleep, alertness, and general well-being, especially among the older shift workers (22).

\section{Study population}

The target group consisted of 403 maintenance workers, 343 of whom were working in a continuous backward-rotating three-shift system. Of the participants, $22 \%$ were inspectors or supervisors, and the rest were mechanics. The participants had to be healthy, permanently employed maintenance workers working in 
the continuous backward-rotating three-shift system. Reasons for exclusion were the existence of any CVD or regular medication for blood pressure, high cholesterol, diabetes, or sleeping disorders. The participants were recruited through advertising at the workplace. The study criteria were described in the advertisements. Altogether 89 men volunteered for the study, all of whom were also eligible for participation. Only three female workers volunteered for the study, which was too few to generate separate groups. Before the study was completed, one participant died accidentally, one changed to fixed night work, one could not take part in the follow-up survey because of sick leave due to a leisure-time injury, and two were on a leave of several months at the follow-up time. Complete data were thus available on 84 male participants, 40 of whom changed to a rapidly forward-rotating shift system, 22 changed to work in a more flexible shift system, and 22 persisted in the old shift system, and thus formed the reference group. The respective dropout rates for the three groups were $0 \%(\mathrm{~N}=0), 12 \%(\mathrm{~N}=3)$, and $8 \%(\mathrm{~N}=2)$. All of the participants were examined by an occupational health physician at the beginning of the study.

All of the participants gave their written informed consent. The Helsinki University Research Ethics Board for Occupational Health reviewed the study plan and gave their approval in advance [record number (Dnro): 158/E2/04; date: 22 June 2004]. The study was performed according to the Helsinki Declaration of 1975, as revised in 1983.

\section{Health outcomes}

Blood tests for serum total cholesterol, HDL cholesterol, LDL cholesterol, triglycerides, fasting plasma glucose (fP-gluc), glycosylated hemoglobin (HbA1c), and highsensitivity C-reactive protein (hs-CRP) were taken after a fasting period of 12 hours. The same trained laboratory assistant drew the blood samples on the first morning shift between 0700 and 0900 , at least 72 hours after the end of the last night shift, at baseline, and at the end of the follow-up phase. All of the blood samples were centrifuged within 2 hours and analyzed on the same day (hs-CRP twice a week) in a certified laboratory according to standard procedures using a Konelab 60i clinical chemistry analyzer (Thermo Fisher Scientific, Ltd, Vantaa, Finland).

Blood pressure and heart rate were measured twice with a fully automatic device (Omron ${ }^{\circledR}$ M4-1, OMRON Healthcare Europe BV, Hoofddorp, Netherlands) after a 5-minute rest in a sitting position. The same trained nurse measured height, weight, and the circumference of the waist and hip according to standard methods. Body mass index (BMI) was calculated (weight/height ${ }^{2}$ ). The participants were advised to avoid smoking and vigorous physical activity at least half an hour before the laboratory tests and clinical measurements.

The self-administered questionnaire contained questions on diseases diagnosed by a physician, regular medication, lifestyle, and dietary factors. Leisure-time physical activity was assessed with the use of a modified version of the International Physical Activity Questionnaire (IPAQ) (28), which requested the number of physical activity sessions per week and minutes per session. The intensity of leisure-time physical activity was inquired about with the aid of examples of strenuous, moderately strenuous, and light physical activity. Daytime sleepiness, the frequency of sleep disturbances, and the probability of falling asleep at work were studied with the Basic Nordic Sleep Questionnaire (BNSQ) (29) and the Epworth Sleepiness Scale (ESS) (30)

To study the effect of shift change on dietary habits, the questionnaire contained 10 questions on the quality of dietary fat (saturated, polyunsaturated), 8 questions on fiber intake (assessed in the diet of the previous day), 8 questions on alcohol consumption, and 1 question on drinks containing caffeine. Sum scales were calculated in order to obtain fat quality (the proportion of unsaturated fats to saturated fats: $>24$ excellent, $<10$ poor) and daily intake of alcohol $(\mathrm{g})$, caffeine $(\mathrm{mg})$, and fibers $(\mathrm{g})$.

The partcipants received the questionnaires at the time of the clinical examinations and were asked to return them in 1 week. The baseline results of the blood tests with the reference values were sent to the participants without comment. Two persons in the old system and one in each of the new shift systems requested dietary counseling for cholesterol. These workers were excluded from all of the statistical analyses when the outcome was waist circumference, waist-to-hip ratio, BMI, blood lipids or glucose, daily alcohol consumption, quality of dietary fat, or the amount of dietary fiber. Occupational status and years in shift work were obtained from the employer's register.

The baseline characteristics of the participants are shown in table 1 . The workers who started in the rapidly forward-rotating shift system were about 10 years older than those who started in the flexible shift system. The levels of CVD risk factors and health habits at baseline are shown in tables 2 and 3 for each study group. Alcohol intake at baseline was the most frequent in the group starting in the rapidly forward-rotating shift system.

\section{Statistical methods}

A repeated-measures analysis of variance (ANOVA) was used for each outcome separately. The variables in the models were time (before and after), shift system (rapidly forward-rotating, flexible, and old shift systems), interaction between time and shift system, age $(<45$, 
Table 1. Baseline characteristics of the participants.

\begin{tabular}{|c|c|c|c|c|c|c|c|c|c|c|}
\hline \multirow[t]{2}{*}{ Study group } & \multirow{2}{*}{$\begin{array}{c}\text { Number } \\
\text { of } \\
\text { participants }\end{array}$} & \multicolumn{3}{|c|}{ Age (years) } & \multicolumn{3}{|c|}{ Occupational status (\%) } & \multicolumn{2}{|c|}{ Work in shifts (years) } & \multirow{2}{*}{$\begin{array}{l}\text { Current } \\
\text { smoking } \\
(\%)\end{array}$} \\
\hline & & Mean & SD & Range & Mechanic & Inspector & Supervisor & Mean & SD & \\
\hline Rapidly forward-rotating shift system & 40 & 47 & 7 & $27-58$ & 92 & 0 & 8 & 23 & 8 & 15 \\
\hline Flexible shift system & 22 & 37 & 9 & $28-58$ & 95 & 0 & 5 & 12 & 6 & 0 \\
\hline Old shift system (control group) & 22 & 44 & 9 & $27-57$ & 54 & 23 & 23 & 21 & 8 & 27 \\
\hline
\end{tabular}

Table 2. Means and standard deviations of the blood tests for cardiovascular risk factors in the study groups before and after the shift change-no statistically significant differences in the interaction between time and group adjusted for age, smoking habits, and alcohol consumption (repeated-measures analysis of variance). (HDL = high-density lipoprotein, $L D L=$ low-density lipoprotein, $f P$-gluc $=$ fasting plasma glycose, $\mathrm{HbAlc}=$ glycosylated hemoglobin, hs-CRP = high-sensitivity C-reactive protein)

\begin{tabular}{|c|c|c|c|c|c|c|c|c|c|c|c|c|c|c|}
\hline \multirow[t]{3}{*}{ Study group } & \multicolumn{6}{|c|}{ Cholesterol ${ }^{\mathrm{a}}$} & \multirow{2}{*}{\multicolumn{2}{|c|}{$\begin{array}{l}\text { Triglycerides a } \\
(\mathrm{mmol} / \mathrm{l})\end{array}$}} & \multirow{2}{*}{\multicolumn{2}{|c|}{$\begin{array}{l}\text { fP-gluc a } \\
(\mathrm{mmol} / \mathrm{l})\end{array}$}} & \multirow{2}{*}{\multicolumn{2}{|c|}{$\begin{array}{l}\mathrm{HbA} 1 \mathrm{c}^{\mathrm{a}} \\
(\%)\end{array}$}} & \multirow{2}{*}{\multicolumn{2}{|c|}{$\begin{array}{l}\text { hs-CRP } \\
\text { (mg/l) }\end{array}$}} \\
\hline & \multicolumn{2}{|c|}{$\begin{array}{c}\text { Total } \\
(\mathrm{mmol} / \mathrm{l})\end{array}$} & \multicolumn{2}{|c|}{$\underset{(\mathrm{mmol} / \mathrm{l})}{\mathrm{HDL}}$} & \multicolumn{2}{|c|}{$\underset{(\mathrm{mmol} / \mathrm{l})}{\mathrm{LDL}}$} & & & & & & & & \\
\hline & Mean & SD & Mean & SD & Mean & SD & Mean & SD & Mean & SD & Mean & SD & Mean & SD \\
\hline \multicolumn{15}{|c|}{ Rapidly forward-rotating shift system $(\mathrm{N}=40)$} \\
\hline Before & 5.3 & 0.8 & 1.4 & 0.3 & 3.3 & 0.8 & 1.4 & 0.9 & 5.3 & 0.6 & 5.2 & 0.3 & 1.2 & 1.6 \\
\hline After & 5.6 & 1.1 & 1.4 & 0.3 & 3.4 & 0.9 & 1.6 & 0.9 & 5.2 & 0.6 & 5.0 & 0.4 & 1.5 & 1.7 \\
\hline \multicolumn{15}{|c|}{ Flexible shift system $(\mathrm{N}=22)$} \\
\hline Before & 5.0 & 0.7 & 1.3 & 0.3 & 3.2 & 0.7 & 1.3 & 0.6 & 5.2 & 0.4 & 5.1 & 0.3 & 0.8 & 0.8 \\
\hline After & 5.0 & 0.8 & 1.4 & 0.3 & 3.1 & 0.8 & 1.3 & 0.7 & 5.0 & 0.4 & 4.9 & 0.4 & 1.1 & 1.2 \\
\hline \multicolumn{15}{|c|}{ Old shift system (control group) ( $\mathrm{N}=22$ ) } \\
\hline Before & 5.2 & 1.1 & 1.5 & 0.3 & 3.0 & 0.9 & 1.2 & 0.9 & 5.2 & 0.4 & 5.1 & 0.2 & 0.9 & 0.6 \\
\hline After & 5.3 & 1.2 & 1.6 & 0.3 & 3.1 & 1.0 & 1.3 & 0.7 & 5.0 & 0.5 & 5.0 & 0.4 & 1.2 & 1.0 \\
\hline
\end{tabular}

a Four persons who received dietary counseling were excluded.

Table 3. Means and standard deviations of the body mass index, waist, waist-to-hip ratio and dietary fat and fiber and the medians and ranges of caffeine and alcohol consumption and leisure-time physical activity in the study groups before and after the shift change-no statistically significant differences in the interaction between time and group adjusted for age, smoking habits, and alcohol consumption (repeated measures analysis of variance).

\begin{tabular}{|c|c|c|c|c|c|c|c|c|c|c|c|c|c|c|c|}
\hline \multirow[t]{2}{*}{$\begin{array}{l}\text { Study } \\
\text { group }\end{array}$} & \multicolumn{2}{|c|}{$\begin{array}{l}\text { Body mass index a } \\
\left(\mathrm{kg} / \mathrm{m}^{2}\right)\end{array}$} & \multicolumn{2}{|c|}{$\begin{array}{l}\text { Waista } \\
\text { (cm) }\end{array}$} & \multicolumn{2}{|c|}{$\begin{array}{l}\text { Waist-to-hip } \\
\text { ratio }^{\text {a }}\end{array}$} & \multicolumn{2}{|c|}{$\begin{array}{l}\text { Quality of } \\
\text { fat }{ }^{a, b}\end{array}$} & \multicolumn{2}{|c|}{$\begin{array}{c}\text { Dietary fibera } \\
\text { (g/day) }\end{array}$} & \multirow{2}{*}{$\begin{array}{c}\begin{array}{c}\text { Caffeine } \\
(\mathrm{mg} / \text { day })\end{array} \\
\text { Median Range }\end{array}$} & \multirow{2}{*}{\multicolumn{2}{|c|}{$\frac{\begin{array}{c}\text { Alcohola } \\
\text { (g/day) }\end{array}}{\text { Median Range }}$}} & \multirow{2}{*}{\multicolumn{2}{|c|}{$\begin{array}{c}\begin{array}{c}\text { Physical activity } \\
\text { (h/week) }\end{array} \\
\text { Median Range }\end{array}$}} \\
\hline & Mean & SD & Mean & SD & Mean & SD & Mean & SD & Mean & SD & & & & & \\
\hline \multicolumn{16}{|c|}{ Rapidly forward-rotating shift system ( $N=40)$} \\
\hline Before & 26 & 3 & 92 & 8 & 0.94 & 0.06 & 16 & 5 & 25 & 11 & $50030-2000$ & 12 & $0-52$ & 2.8 & $0-1$ \\
\hline After & 26 & 3 & 94 & 8 & 0.96 & 0.06 & 17 & 6 & 23 & 11 & $400 \quad 0-1760$ & 11 & $0-47$ & 2.8 & $0-8$ \\
\hline \multicolumn{16}{|c|}{ Flexible shift system ( $\mathrm{N}=22)$} \\
\hline Before & 27 & 3 & 90 & 6 & 0.91 & 0.04 & 17 & 4 & 21 & 10 & $450 \quad 0-1220$ & 5 & $1-40$ & 2.6 & $0-12$ \\
\hline After & 27 & 3 & 93 & 6 & 0.93 & 0.04 & 18 & 4 & 22 & 7 & $500 \quad 0-1600$ & 7 & $0-49$ & 2.5 & $0-1$ \\
\hline \multicolumn{16}{|c|}{ Old shift system (control group) ( $\mathrm{N}=22$ ) } \\
\hline Before & 25 & 2 & 91 & 7 & 0.93 & 0.05 & 15 & 6 & 22 & 10 & $38090-1160$ & 5 & $0-20$ & 3.8 & $0-8$ \\
\hline After & 25 & 2 & 92 & 8 & 0.93 & 0.05 & 15 & 7 & 22 & 10 & $35030-1100$ & 4 & $0-22$ & 3.5 & $0-21$ \\
\hline
\end{tabular}

a Four persons who received dietary counseling were excluded.

b Proportion of unsaturated fats to saturated fats.

c At least moderately strenuous.

$\geq 45$ - years) at baseline, smoking (yes, no) at baseline, and alcohol consumption ( $\leq 1,2, \geq 3$ alcohol doses daily) at baseline and at the end of the study. Smoking was adjusted at baseline because it was considered to have long-term effects on the outcomes. Alcohol consumption was adjusted at the same point of time as the outcome because it was considered to have mainly short-term effects on the outcomes. The effects of two new shift systems were evaluated in this study. Therefore, the same models containing three-shift systems were also 
used to test whether the rapidly forward-rotating shift system differed from the old shift system and whether the flexible shift system differed from the old shift system. All of the adjusted P-values presented in this article are P-values of the interaction term for time and shift system (three or two shift systems). The distribution of caffeine and alcohol consumption and physical activity was skewed. Ranks were used instead of original values for these outcomes in the analysis of variance. All of the statistical analyses were calculated using the Statistical Analysis System (SAS, version 9.1, SAS Institute Inc, Cary, NC, USA, 2004).

\section{Results}

The studied interventions influenced the shift workers' alertness. The mean number of days per week on which the workers reported sleepiness decreased in the group with the rapidly forward-rotating shift system, but increased slightly in the other two groups (figure 1). Daytime sleepiness showed a declining trend when all three shift systems were compared with each other $(\mathrm{P}=0.06)$. In further analyses, the changes in daytime sleepiness differed significantly between the forward-rotating and the old shift systems $(\mathrm{P}=0.02)$.

When assessed on the Epworth Sleepiness Scale, the probability of falling asleep at work decreased slightly in the rapidly forward-rotating shift system, from 6.4 (SD 2.7) to 6.2 (SD 3.5), and in the flexible shift system, from 7.5 (SD 5.3) to 7.1 (SD 4.4), whereas in the old shift system the Epworth Sleepiness Scale increased, from 7.8 (SD) 3.8 to 8.3 (SD 3.9). These changes in

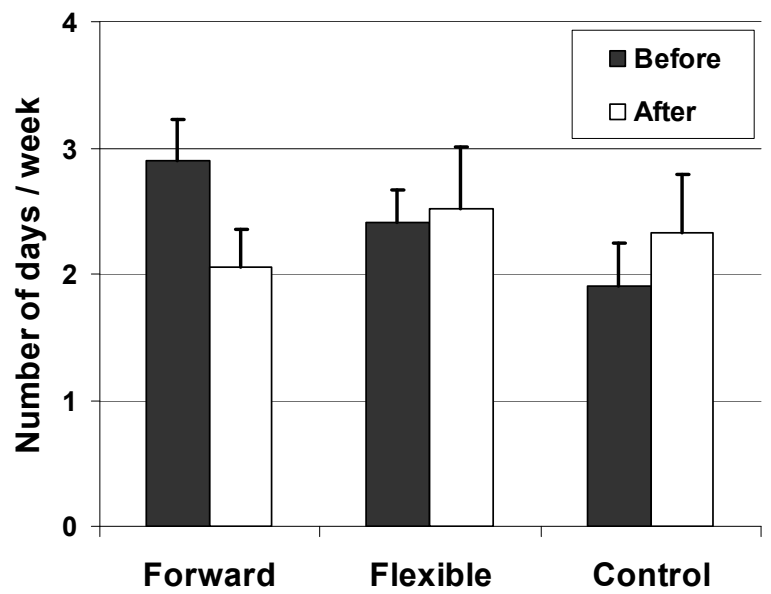

Figure 1. Effect of a change of shifts on the number of days/week (mean and standard error of the mean) with sleepiness in the group with a rapidly forward-rotating shift system (Forward, $\mathrm{N}=40$ ), the group with a more flexible shift system (Flexible, $\mathrm{N}=22$ ), and the group with the old shift system (Control, $\mathrm{N}=22$ ). All of the groups started with a slowly backward-rotating shift system with no flexibility. the ESS scores were not statistically significant. The new shift systems did not change the frequency of sleep disturbances.

The studied changes in the shift system influenced blood pressure and heart rate (figure 2). The changes in systolic blood pressure differed between the three study groups $(\mathrm{P}=0.049)$. The mean systolic blood pressure decreased by $6 \mathrm{~mm} \mathrm{Hg}$ in the group with the flexible shift system, increased by $2.5 \mathrm{~mm} \mathrm{Hg}$ in the group with the rapidly forward shift system, but remained unchanged in the old shift system. The changes in diastolic blood pressure were not significant. When the three study groups were compared, the changes in heart rate had a decreasing trend $(\mathrm{P}=0.06)$, and this trend persisted
Flexible
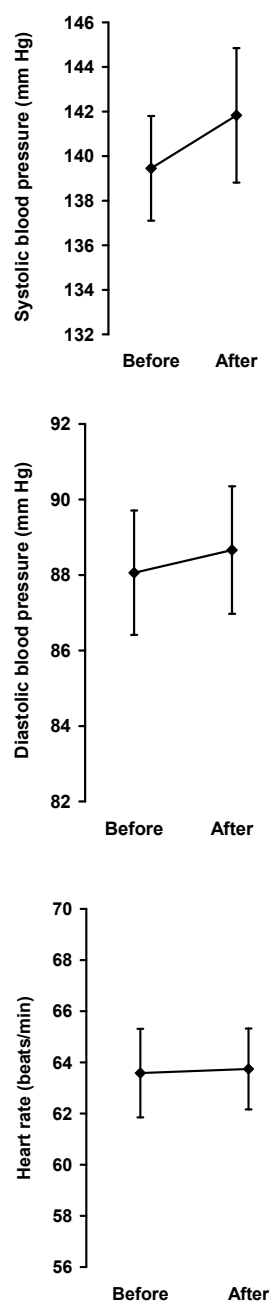
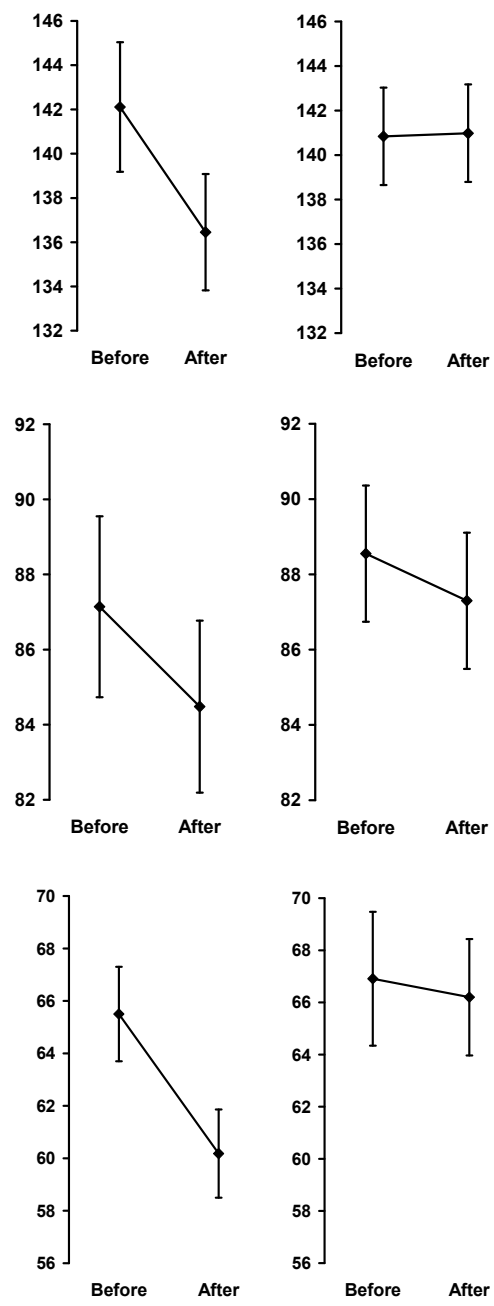

Figure 2. Effect of a change of shifts on systolic blood pressure, diastolic blood pressure, and heart rate (mean and standard error of the mean) in the group with a rapidly forward-rotating shift system (Forward, $\mathrm{N}=40$ ), the group with a more flexible shift system (Flexible, $\mathrm{N}=22$ ), and the group with the old shift system (Control, $\mathrm{N}=22$ ). All of the groups started with a slowly backward-rotating shift system with no flexibility. 
when the flexible shift system was compared with the old system in further analyses $(\mathrm{P}=0.06)$.

The new shift systems did not affect the other measured CVD risk factors during the follow-up (table 2). BMI, waist circumference, or waist-to-hip ratio did not change across the time period in the three study groups. There were no statistically significant changes between the groups with respect to leisure-time physical activity or dietary habits as assessed by the quality of fat or the intake of fiber, caffeine, or alcohol (table 3).

\section{Discussion}

In our study, the change from a slower backward-rotating shift system (shift changes every three shifts) to a very quickly forward-rotating shift schedule improved the perceived alertness of the workers, even though the operating hours at night actually increased. An interesting finding was the decrease in the workers' systolic blood pressure and a decreasing trend in heart rate in the shift system in which the individual flexibility of workhours was combined with company-based operational flexibility.

The observed beneficial effect of a rapidly forwardrotating shift system on workers' alertness is consistent with the finding of our earlier reports $(22,23)$, and a study report by de Valck et al (24). The declining trends in the ESS score and caffeine consumption in this group were congruent with the decrease in daytime sleepiness.

The intervention from a rather slow backward-rotating system to a very rapidly forward-rotating shift system consisted of changes in both the number of consecutive morning, evening, and night shifts (from three to one) and in the direction of the rotation (from backwards to forwards), as well as of some minor changes in the starting and ending of the shifts. However, the number of successive shifts (three) and free days (two) did not change. The observed results in this group can probably not be connected to any single element of the shift schedule, merely to the combination of the simultaneous changes.

The older workers benefited from a rapidly forwardrotating shift schedule in our earlier studies $(22,23)$, one of which was carried out earlier with the same line maintenance unit as in our present study. This finding is probably due to a slower circadian adaptation to night shifts and the increased "morningness" of older workers. With only a single night shift, no delay in the circadian rhythms is necessary, and morningness or an inability to shift circadian rhythms during evening and night shifts should support the recovery of sleep-wakefulness during the following free days. The participants slept a shorter time during the day after a single night shift, but longer and better during the recovery period between the single night and morning shifts (22). Because of earlier positive experiences gained by older shift workers in the rapidly forward-rotating shift system, a higher number of older persons was selected for this group in our current study.

A decrease of 10 or even $2 \mathrm{~mm} \mathrm{Hg}$ in systolic blood pressure has been shown to be associated with a marked reduction in the risk of stroke (31) and mortality from ischemic heart disease (16). The observed $6 \mathrm{~mm} \mathrm{Hg}$ decrease of systolic blood pressure, if long-lasting, may thus have a beneficial impact on workers' health. The decrease in systolic blood pressure in combination with a decreasing trend for heart rate in the group with the more flexible shift system may reflect a relief of psychophysiological stress. According to our knowledge, there are no earlier studies on the effects of flexible shift systems on psychophysiological stress reactions. In one short-term study, systolic blood pressure and the urinary excretion of catecholamines decreased after a 4-week period of clockwise, as compared with counter-clockwise, shift rotation (20). Another study with a 3-week follow-up period found no difference in salivary cortisol levels between a fast-forward and a slow-backward shift system (24).

A chronic situation of sustained psychophysiological activation and incomplete recovery has been proposed to form a pathway to chronic health impairment (32). According to cross-sectional data from the Third EU Survey on Working Conditions (25), low individual flexibility and high variability (ie, company-based flexibility) in workhours was associated with a higher prevalence of health problems (eg, sleeping problems, overall fatigue, anxiety, heart disease), while low variability in workhours combined with high autonomy had a positive association with workers' health and well-being. Flexible workhours increase job satisfaction (33), and the worker's own satisfaction with the workhours indicates tolerance to the debilitating effects of shift work on health, at least for women (34). Adequate control of worktime was also associated with fewer negative effects of long total workhours (work + home) on subjective health and sickness absence (26).

The change from a fixed backward-rotating shift system to a more flexible shift schedule basically consisted of a change in the number of consecutive days off between evening, morning, and night shifts (from two to three). However, the varying lengths of the morning and evening shifts also made the shift system more flexible. Within the limits of the work contract, this shift system enabled individual and company-based arrangements of workhours. The employer could change the shift lengths and increase the number of successive evening and morning shifts from three to four by rescheduling 
"free" days in case of altered operational needs. The employees were also able to change individual shifts and extend duty off periods to better adapt their workhours to their private needs.

The decrease in systolic blood pressure in combination with the declining trend for heart rate probably indicates a decrease in psychophysiological stress in the more flexible shift system. It is possible that the increase in the individual flexibility of the shift system decreased the psychophysiological stress of the workers. Unfortunately, the questionnaire used in this study did not include items on workers' opinions of work stress or their opportunities to influence their workhours. In the flexible shift system, the actual time-off period from the end of the last morning shift to the beginning of the first night shift also varied in a wider range (from 52.5 to 80.5 hours) than in the other two shift systems. It is thus possible that the improvement in psychophysiological stress could also be explained by the longer recovery time between the separate shift sequences.

The waist-to-hip ratio, BMI, blood lipids, glycemic control measured by HbA1c, and fasting glucose did not change. The dietary and lifestyle factors remained stable in all three groups during the study. There were no changes in the quality of fat or in the amount of fiber intake. Neither alcohol consumption nor leisure-time physical activity changed. Data on dietary habits and alcohol consumption were collected with a structured questionnaire. Food records were not used because food recording is a laborious method, and we wanted to ensure the highest possible participation rate. We managed to attain this goal, as the total participation rate was $100 \%$.

With reference to the generalization of the results, our intervention study, conducted in field conditions, had certain limitations. Since more than one shift characteristic changed simultaneously, it is not possible to conclude which component of the new shift system accounted for the positive results. To predict the effect of a similar shift system change in other worksites, the intervention would have to be designed in a similar way. The participation rate of our study was low. In addition, we could not randomize the allocation of the participants into the study groups, but we could control for the differences in age, smoking habits, and alcohol intake in the statistical analyses. The educational and socioeconomic background of the participants, regardless of current occupational status, was homogeneous, and the work conditions were similar between the groups. The study protocol was well standardized, we used a control group, and the dropout rate was low, all of which contribute to the reliability of the data and results. However, due to the short follow-up time and small study groups, the results may underestimate the health outcomes of the shift changes. For instance, the reduction of daytime sleepiness by about 1 day a week is probably clinically important. In addition, the short follow-up time possibly impeded the detection of permanent changes in CVD risk factors due to the change in shifts.

In conclusion, our findings on the alertness of shift workers in this study are consistent with those of our previous studies. A faster speed of rotation, together with a change to a forward direction in shift rotation alleviates daytime sleepiness. A possible new finding is that combining individual elements with company-based flexibility in a shift system may have favorable effects on the psychophysiological stress of shift workers.

\section{Acknowledgments}

The authors are grateful to the representatives of the employer, the employees of Finnair Line Maintenance, and the staff of Finnair Health Services. We thank M Aittasalo from the UKK Institute for Health Promotion Research for her assistance with the estimation of leisure-time physical activity.

The study was supported by a grant from the Mutual Pension Insurance Company Ilmarinen.

\section{References}

1. Åkerstedt T. Shift work and disturbed sleep/wakefulness. Occup Med. 2003;53:89-94.

2. Knutsson A. Health disorders of shift workers. Occup Med. 2003;53:103-8.

3. Karlsson B, Alfredsson L, Knutsson A, Andersson E, Torén K. Total mortality and cause-specific mortality of Swedish shiftand dayworkers in the pulp and paper industry in 1952-2001. Scand J Work Environ Health. 2005;31(1):30-5.

4. Fujino Y, Iso H, Tamakoshi A, Inaba Y, Koizumi A, Kubo T, et al. A prospective cohort study of shift work and risk of ischemic heart disease in Japanese male workers. Am J Epidemiol. 2006;164:128-35.

5. Tenkanen L, Sjöblom T, Kalimo R, Alikoski T, Härmä M. Shift work, occupation and coronary heart disease over 6 years of follow-up in the Helsinki Heart Study. Scand J Work Environ Health. 1997;23(4):257-65.

6. Bøggild H, Knutsson A. Shift work, risk factors and cardiovascular disease [review]. Scand J Work Environ Health. 1999;25(2):85-99.

7. Knutsson A, Åkerstedt T, Jonsson BG. Prevalence of risk factors for coronary artery disease among day and shift workers. Scand J Work Environ Health. 1988;14:317-21.

8. Karlsson BH, Knutsson AK, Lindahl BO. Is there an association between shift work and having a metabolic syndrome? results from a population based study of 27485 people. Occup Environ Med. 2001;58:747-52.

9. Karlsson BH, Knutsson AK, Lindahl BO, Alfredsson LS. Metabolic disturbances in male workers with rotating threeshift work: results of the WOLF study. Int Arch Occup Environ 
Health. 2003;76:424-30.

10. Sookoian S, Gemma C, Fernandez Gianotti T, Burgueño A, Alvarez A, González CD, et al. Effects of rotating shift work on biomarkers of metabolic syndrome and inflammation. J Intern Med. 2007;261:285-92.

11. Ghiasvand M, Heshmat R, Golpira R, Haghpanah V, Soleimani A, Shoushtarizadeh P, et al. Shift working and risk of lipid disorders: a cross-sectional study. Lipids Health Dis. 2006;5:9.

12. Sakata K, Suwazono Y, Harada H, Okubo Y, Kobayashi E, Nogawa K. The relationship between shift work and the onset of hypertension in male Japanese workers. J Occup Environ Med. 2003;45:1002-6.

13. Morikawa Y, Nakagawa H, Miura K, Soyama Y, Ishizaki M, Kido T, et al. Effect of shift work on body mass index and metabolic parameters. Scand J Work Environ Health. 2007;33(1):45-50.

14. Peter R, Alfredsson L, Knutsson A, Siegrist J, Westerholm P. Does a stressful psychosocial work environment mediate the effects of shift work on cardiovascular risk factors? Scand J Work Environ Health. 1999;25(4):376-81.

15. Oishi M, Suwazono Y, Sakata K, Okubo Y, Harada H, Kobayashi $\mathrm{E}$, et al. A longitudinal study on the relationship between shift work and the progression of hypertension in male Japanese workers. J Hypertens. 2005;23:2173-8.

16. Lewington S, Clarke R, Qizilbash N, Peto R, Collins R. Agespecific relevance of usual blood pressure to vascular mortality: a meta-analysis of individual data for one million adults in 61 prospective studies. Lancet. 2002;360:1903-13.

17. Virtanen S, Notkola V. Socioeconomic inequalities in cardiovascular mortality and the role of work: a register study of Finnish men. Int J Epidemiol. 2002;31:614-21.

18. Härmä M. Workhours in relation to work stress, recovery and health [review]. Scand J Work Environ Health. 2006;32(6, special issue):502-14.

19. Driscoll TR, Grunstein RR, Rogers NL. A systematic review of the neurobehavioural and physiological effects of shiftwork systems. Sleep Med Rev. 2007

20. Orth-Gomer K. Intervention on coronary risk factors by adapting a shift work schedule to biologic rhythmicity. Psychosom Med. 1983;45:407-415.

21. Bøggild H, Jeppesen HJ. Intervention in shift scheduling and changes in biomarkers of heart disease in hospital wards. Scand J Work Environ Health. 2001;27(2):87-96.

22. Härmä M, Hakola T, Kandolin I, Sallinen M, Vahtera J, Bonnefond A, et al. A controlled intervention study on the effects of a very rapidly forward rotating shift system on sleep-wakefulness and well-being among young and elderly shift workers. Int J Psychophysiol. 2006;59:70-79.

23. Hakola T, Härmä M. Evaluation of a fast forward rotating shift schedule in the steel industry with a special focus on ageing and sleep. J Hum Ergol (Tokyo). 2001;30:315-9.

24. De Valck E, Quanten S, Berckmans D, Cluydts R. Simulator driving performance, subjective sleepiness and salivary cortisol in a fast-forward versus a slow-backward rotating shift system. Scand J Work Environ Health. 2007;33(1):51-7.

25. Costa G, Åkerstedt T, Nachreiner F, Baltieri F, Carvalhais J, Folkard S, et al. Flexible working hours, health, and well-being in Europe: some considerations from a SALTSA project. Chronobiol Int. 2004;21:831-44.

26. Ala-Mursula L, Vahtera J, Kivimäki M, Kevin MV, Pentti J. Employee control over working times: associations with subjective health and sickness absences. J Epidemiol Community Health. 2002;56:272-8.

27. Evans K, Laker MF. Intra-individual factors affecting lipid, lipoprotein and apolipoprotein measurement: a review. Ann Clin Biochem. 1995;32 ( Pt 3):261-80.

28. Craig CL, Marshall AL, Sjöstrom M, Bauman AE, Booth ML, Ainsworth BE, et al. International physical activity questionnaire: 12-country reliability and validity. Med Sci Sports Exerc. 2003;35:1381-95.

29. Partinen M, Gislason T. Basic Nordic Sleep Questionnaire (BNSQ): a quantitated measure of subjective sleep complaints. J Sleep Res. 1995;4:150-5.

30. Johns MW. A new method for measuring daytime sleepiness: the Epworth sleepiness scale. Sleep. 1991;14:540-5.

31. Lawes CM, Bennett DA, Feigin VL, Rodgers A. Blood pressure and stroke: an overview of published reviews. Stroke. 2004;35:1024.

32. Geurts SAE, Sonnentag S. Recovery as an explanatory mechanism in the relation between acute stress reactions and chronic health impairment. Scand J Work Environ Health. 2006;32(6, special issue):482-92.

33. Costa G, Sartori S, Åkerstedt T. Influence of flexibility and variability of working hours on health and well-being. Chronobiol Int. 2006;23:1125-37.

34. Axelsson J, Lowden A, Kecklund G. Recovery after shift work: relation to coronary risk factors in women. Chronobiol Int. 2006;23:1115-24.

Received for publication: 17 June 2007 